\title{
Ciencias Sociales y Politieas Públieas en Chile: qué, cómo y para qué se investiga en el Estado
}

\author{
MANUEL ANTONIO GARRETÓN" \\ MARÍA ANGÉLIGA GRUZ \\ FRANCISCO ESPINOZA*
}

\section{Resumen}

En este artículo se presentan algunos de los resultados de un estudio sobre los vínculos entre la investigación de las ciencias sociales y la formulación y gestión de las políticas públicas en el Chile de la post transición democrática. A partir de una muestra de investigaciones realizadas en los ministerios, clasificados en tradicionales, nuevos, y políticos, se estudian cuatro cuestiones: a) quiénes y en qué condiciones investigan para el Estado, b) contenido de las investigaciones c) perspectivas metodológicas d) usos de las investigaciones. Las respuestas a estas cuestiones permiten plantear algunas conclusiones en torno a dos aspectos. Por el lado del Estado, qué efectos tienen estos estudios para la calidad de las políticas públicas en el contexto de los debates sobre la calidad de la democracia en Chile. Y por el lado de las ciencias sociales, cómo afecta el desarrollo de las disciplinas - particularmente para la sociología- la presencia del Estado como uno de los principales demandantes de conocimiento científico aplicado.

Palabras-clave: Investigación. Políticas públicas. Sociología. Conocimiento experto. Estado. Uso del conocimiento.

\footnotetext{
* Doctor en Sociología. Ecole des Hautes Etudes en Sciences Sociales, Paris. Profesor Titular Depto de Sociología Universidad de Chile.

** PhD (c) Universidad Complutense de Madrid, Docente e Investigadora de la Carrera de Sociología, Universidad de Valparaíso.

*** Licenciado en Sociología, Asistente de Investigación, Universidad de Valparaíso.
} 


\section{Presentación}

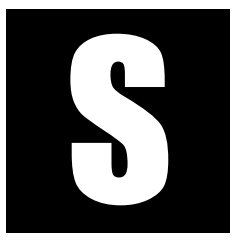

i bien la relación entre ciencia y Estado puede abordarse desde múltiples aristas, en este artículo nos interesa profundizar en sólo una de ellas: los vínculos entre la investigación de las ciencias sociales y la formulación y gestión de las políticas públicas en el Chile de la post transición democrática. Desde la recuperación de la democracia a inicios de la década del 90', en los gobiernos de la Concertación ${ }^{1}$ diversas instancias del aparato público chileno, dependientes de los distintos Ministerios, vienen realizando o encargando estudios e investigaciones en el área de las ciencias sociales, con el objetivo de que sus resultados sirvan de insumo a las políticas públicas. Ello ha operado en el contexto de la llamada modernización del Estado.

Este proceso ocurre en un marco político y teórico-ideológico en el cual se incentiva que las políticas públicas progresivamente se orienten por criterios técnicos más que únicamente políticos, donde los técnicos debieran apoyarse entre otros, en la información rigurosa y sistemática derivada de los conocimientos expertos.

Por otra parte, el desarrollo de la producción de las ciencias sociales en Chile, tradicionalmente se ha analizado con relación a los campos más académicos, Universidades y Centros de Investigación, con menor visibilidad del aparato público estatal en cuanto ámbito de producción y uso de un conocimiento que contribuye a los desarrollos disciplinarios a través de su complejización.

1 Sobre la democratización política chilena y los gobiernos de la coalición de centro-izquierda gobernante desde 1990, conocida como Concertación de Partidos por la Democracia, ver Garretón (2007b). 
En el cruce de estos espacios -las políticas públicas, por una parte, y desarrollo de las ciencias sociales, por el otro- realizamos un estudio orientado al análisis de la investigación en ciencias sociales, particularmente la sociología, aplicadas a las políticas públicas. El principal objetivo de este proyecto era conocer las características de tales investigaciones para el caso de las ciencias sociales - entre 1992 y 2005 - y el uso dado a ellas por el Estado. Como antecedente reciente de este tipo de estudio en Chile, puede indicarse el estudio de Ramos que indaga en cómo investigan los sociólogos a principios del siglo XXI (RAMOS, 2005). Se trata de resultados referidos a las cuestiones disciplinarias, los equipos de investigación y los aspectos teóricos y metodológicos que caracterizan a los estudios. Nuestro propósito es ampliar esta temática refiriéndola, por una parte a las investigaciones relacionadas con las políticas públicas y, por otra, al desarrollo de la sociología.

En este artículo presentaremos algunos resultados de la primera fase del proyecto ${ }^{2}$, referidos a las características de las investigaciones estudiadas, a partir de cuatro preguntas básicas: a) quiénes y en qué condiciones investigan para el Estado, b) qué es lo que mayoritariamente se estudia, c) cómo se investiga y d) para qué se hace. Las respuestas a estas interrogantes nos permiten plantear algunas conclusiones en torno a dos aspectos. Por el lado del Estado, qué efectos tienen estos estudios para la calidad

\footnotetext{
2 Se trata del Proyecto FONDECYT № 1070966. En él participaron como investigadores Manuel Antonio Garretón (Departamento de Sociología, Facultado de Ciencias Sociales Universidad de Chile) y María Angélica Cruz (Carrera de Sociología- CIS, Instituto de Historia, Facultad de Humanidades, Universidad de Valparaíso). El proyecto fue realizado en los años 2007-2008 y participaron, además, los asistentes de investigación Macarena Orchard y Francisco Espinoza, las tesistas María Graciela López y Paulina Pavez, la consultora Claudia Gutiérrez y el personal de apoyo formado por Juan Pablo Rodríguez, Francisco Zamorano y Carolina Grimaldi. La primera fase del proyecto del que extraemos los resultados para este artículo consistió en un estudio general cuantitativo de las características de las investigaciones en varios Ministerios. La segunda fase en etapa de conclusión consistió en un estudio cualitativo a través de entrevistas en profundidad de dos Ministerios: Educación y Servicio Nacional de la Mujer. Agradecemos la colaboración bibliográfica proporcionada por Ingrid Sarti.
} 
de las políticas públicas en el contexto de los debates sobre la calidad de la democracia en Chile. Y por el lado de las ciencias sociales, cómo afecta el desarrollo de las disciplinas - particularmente para la sociología- la presencia del Estado como uno de los principales demandantes de conocimiento científico aplicado.

\section{Antecedentes metodológicos}

El estudio en el que nos basamos es de carácter exploratorio y mezcla los diseños cuantitativos y cualitativos en dos etapas. En la primera, utilizamos una estrategia de investigación social cuantitativa, analizando una muestra de 210 investigaciones realizadas (encargadas o ejecutadas) por alguna unidad ministerial, las que fueron leídas y codificadas en diversas variables (Ej. marcos teóricos y metodológicos, tipo de ejecutores, objetivos, etc.) para luego ser analizadas estadísticamente. La muestra de ministerios seleccionada se clasificó en tres categorías: a) la que corresponde a lo que puede denominarse orientaciones tradicionales de la política pública, que para simplificar en adelante llamaremos política tradicional o clásica, que agrupa las unidades ministeriales vinculadas a los temas sectoriales como trabajo, educación, vivienda y salud, y que en la muestra está representada por la DIRTRAB (Dirección del Trabajo), MINEDUC (Ministerio de Educación) y MINVU (Ministerio de Vivienda y Urbanismo); b) la que corresponde a las orientaciones emergentes de la denominada nueva política, que incluyen los temas de género, participación, pobreza, cultura, medio ambiente, representados en la muestra por SERNAM (Servicio Nacional de la Mujer), DOS (División de Organizaciones Sociales del Ministerio Secretaría General de Gobierno) y MIDEPLAN (Ministerio de Planificación) y c) la que corresponde a la orientación política de la gestión gubernamental que en adelante aparecerán como minis- 
terio político gubernamental, representado en la muestra por la SEGPRES (Ministerio Secretaria General de la Presidencia).

\section{Conocimiento, investigación sociológica y políticas públicas}

Nuestro trabajo buscaba conocer las características de las investigaciones realizadas por el Estado correspondientes a las diversas disciplinas de las ciencias sociales pero desde una perspectiva de análisis sociológica. Particularmente hemos buscado articular herramientas de la sociología política y la sociología de la ciencia, con el trasfondo más amplio de nuestra preocupación por los desafíos epistemológicos y políticos que cubren las actuales relaciones entre ciencia y política.

Es evidente que los procesos políticos tienen ritmos y requerimientos distintos a los de los procesos investigativos y, en general, son más acelerados en la búsqueda de resultados y legitimación social. En este sentido la relación entre ambos procesos, especialmente, en el campo de las políticas públicas no es directa, en el sentido que no puede pensarse que éstas últimas sean resultado de las primeras y que también es difícil que en la formulación y gestión de las políticas públicas se contemple explícitamente la investigación. Esta, por su parte, está condicionada por las posibilidades de financiamiento provenientes tanto del Estado como de los fondos privados nacionales o, en menor medida, extranjeros ${ }^{3}$. A su vez esta dependencia de la comunidad científica de los fondos públicos - que no es una situación nueva ni característica sólo del contexto chileno, sino que es un elemento clave de la relación entre la ciencia y el

\footnotetext{
3 En relación con el financiamiento, de acuerdo a un estudio, un $67 \%$ de los encuestados señala que los recursos para las investigaciones son insuficientes y un $27 \%$ opina que son muy insuficientes. Al clasificar las fuentes de financiamiento indicadas por los encuestados, el $35 \%$ de los fondos reconocidos son públicos. Las otras fuentes de financiamiento son las universidades (19\%), los organismos internacionales (15\%) y las fundaciones privadas (13\%) (ANSOLEAGA, 2005).
} 
gobierno desde el siglo XIX, y con mayor fuerza en el siglo XX- genera una doble interacción: para los científicos, los poderes públicos son sinónimos de recursos y de reconocimiento institucional, y para los gobernantes los científicos son fuentes de peritaje y legitimación (IHL; KALUSZYNSKI, 2002), por lo que aquello que se investigue depende en gran medida de los ámbitos en que el gobernante desee peritaje o legitimación.

Vale la pena recordar que los problemas públicos no siguen un esquema lineal basado sólo en juicios "objetivos" externos a los sujetos, sino que su identificación y selección responde también a elementos subjetivos (MENY; THOENIG, 1992). Frente a esta cuestión, la investigación tiene un rol técnico en tanto provee de "datos", pero también una dimensión política dado que el conocimiento generado puede ser parte de batallas por la definición de lo que debiera o no ser materia de injerencia pública. Desde las epistemologías feministas, por ejemplo, se está abriendo el debate sobre la necesidad de mostrar que la investigación científica participa de las redes, debates y prácticas que van configurando la realidad - en tanto la realidad se concibe como socialmente construida - argumentando que no hay posibilidad de generar conocimientos universales y neutros sino que más bien éstos son conocimientos situados (HARAWAY, 1995). Bajo esta premisa, podríamos sostener que la investigación que se realiza desde y para el Estado, cuando se pretende universal y neutra, podría ser peligrosamente utilizada como un elemento de juicio "objetivo" en la definición de un problema público, oscureciendo el legítimo y necesario carácter intersubjetivo, y por tanto político, que rodea la definición -y aceptación- de un problema como problema público y por tanto objeto de políticas.

En el año 2006, cerca de 2000 participantes de 80 países se reunieron en Rosario, Argentina, para reflexionar sobre los vínculos entre políticas públicas y ciencias sociales (Foro Internacional sobre el Nexo entre Políticas y Ciencias Sociales, 2006). Una de las señales más claras que 
allí se emitieron fue apelar a que las políticas públicas se sustentaran en investigaciones de las ciencias sociales. Allí se indicaron algunos posibles ejes de articulación entre las ciencias sociales y las políticas públicas: a) la relación entre la información y la gestión de proyectos que puede concretarse con la investigación-acción; b) la identificación y comunicación de experiencias en casos análogos de las mejores prácticas para enriquecer cómo construir las decisiones políticas de consenso; y c) la relación entre la información y la evaluación de las políticas públicas. En otras palabras, se apelaba a que la investigación social apoye la toma de decisiones durante todo el ciclo de la política pública.

Si nos preguntamos por la relación entre ciencias sociales y políticas públicas en Chile, puede afirmarse que la economía ha sido la primera ciencia social enseñada a nivel universitario en materia de políticas públicas. En esta disciplina, no obstante contar con diversos marcos referenciales, predomina la visión neoclásica (neoliberalismo) a la hora de aplicar el conocimiento de las ciencias sociales a la formulación de las políticas públicas (URZÚA, 2006). La sociología, no ha logrado hasta la fecha tener el grado de injerencia que tiene la economía en el proceso de formulación de las políticas. Situación similar ocurre con la ciencia política que sólo a partir de la década de los noventa cobra cierta relevancia. Pareciera ser que en el ámbito estatal la economía ha logrado conservar su estatus de consejera privilegiada en base a la imposición de un "régimen de objetividad" (IHL; KALUSZYNSKI, 2002), lo que lleva a preguntarse por las causas que llevaron a esta disciplina a presentarse ante el Estado y la comunidad científica como la ciencia social más adecuada para tomar las decisiones en el Estado, lo que sería materia de otra investigación.

No obstante, el proceso de redemocratización en Chile -así como en otros países de Latinoamérica- instala condiciones de posibilidad para que la sociología vuelva a participar en el Estado, pero que en este con- 
texto cambia su cariz. En efecto, desde su institucionalización y hasta la dictadura militar, la sociología nutrió conceptualmente parte de los proyectos gubernamentales vinculados, aunque en cierta tensión, con los proyectos académicos (GARRETÓN, 2007a). Sin embargo, es en el período post-autoritario cuando la sociología se reinserta en el Estado a través del uso que se hace de la investigación científico social en los procesos de diseño y desarrollo de las políticas públicas.

A inicios de los 90', Brunner y Súnkel (1993), señalaban que los investigadores sociales latinoamericanos -o al menos un sector de ellosformarían parte una emergente categoría de analistas que estarían en "... disposición de producir, transportar, usar y aplicar conocimientos para la identificación, resolución y arbitraje de problemas; (y que tendrían) la habilidad de manipular conocimientos para el cumplimiento de esas funciones" (BRUNNER; SÚNKEL, 1993, p. 12). Éstos - a diferencia de los funcionarios estatales - por su conciencia sobre la relación entre conocimiento y poder, su convicción acerca de que poseen conocimientos relevantes para el ejercicio efectivo del poder, y acostumbrados a la marginación y al disenso, intentarían mantener una cierta distancia crítica respecto de otros actores que también se insertan en el Estado, aunque con otros saberes menos específicos en relación a los campos o áreas de acción. Los investigadores procurarían establecer una lógica de interacción con las estructuras jerárquicas de gobierno, más que una lógica de subordinación u obediencia (BRUNNER; SÚNKEL, 1993). Sin embargo, podemos relativizar esa convicción si se suman otros análisis más recientes sobre la forma en que la investigación social se vincula con las políticas públicas, los cuales señalan que hay diversos modelos de aplicación del conocimiento a tales políticas, como veremos más adelante (AURIAT, 1997).

En Chile y, en general en América Latina, puede decirse que la sociología desde sus orígenes ha producido un tipo de conocimiento más 
centrado en "la sociedad histórica concreta" que en "lo social abstracto" (GARRETÓN, 1994; VASCONI, 1996), y que hoy enfrenta un cierto cuestionamiento de su objeto de estudio debido a los procesos de globalización, individualización y explosiones identitarias, que parecieran hacer estallar la idea misma de "sociedad" y debilitar la idea de un proyecto intelectual crítico de la sociología.

Así, si retomamos una de las periodizaciones propuestas para el análisis de la sociología en Chile (GARRETÓN, 2005), observaremos que la vinculación entre la disciplina y la política ha sido una constante, incluido el período autoritario, aunque las formas de esa relación hayan cambiado significativamente. A la primera etapa de creación, institucionalización y profesionalización de la disciplina (mediados de los 50' hasta 1973) contribuyó el clima de una sociedad que se sentía en profunda transformación, ligada los procesos de modernización y democratización progresiva que rompían con la dominación oligárquica. Así, se reconocía la necesidad de contar con un tipo de experto encargado de conocer lo nuevo y de proponer alternativas. Asimismo, había un alto grado de legitimidad respecto de la reflexión sobre la práctica histórica de una sociedad, reivindicando para las ciencias sociales un carácter de "conciencia" de los proyectos socio-istóricos y su inserción en ellos para la formulación de su visión ideológica y programática. Tras la institucionalización incipiente con la creación de escuelas de sociología (1950-60), vino la consolidación disciplinaria que terminó en una fuerte radicalización ideológica (1960-1973), asociada, aunque mediatamente, a la polarización política del país. Como es corriente afirmarlo, durante esta etapa se sucedieron dos modelos: la "sociología científico-profesional", articulada en torno a la modernización científica y la especialidad disciplinaria; y la "sociología científico-crítica", que giró en función del rol crítico de las ciencias sociales. El paso de un modelo a otro no fue independiente del contexto social. El cambio de modelos trajo aparejado un cambio en la 
inserción profesional de los científicos sociales: en el primero primaban los investigadores que se desempeñaban en la academia y el Estado; mientras que en la sociología crítica hay una diferenciación de las formas de inserción social, desde el investigador académico, el experto en nuevos problemas sociales o el tecnócrata, hasta el intelectual y el ideólogo militante. Además, con el cambio de modelos y el uso de enfoques más integrados se crearon diversos centros interdisciplinarios de investigación, siempre en el marco institucional de las Universidades, sobre áreas problemas o análisis globales de la sociedad, contribuyendo a disminuir el rezago de la investigación.

Tras el golpe de Estado de 1973 se desarrolla una segunda etapa en la evolución de la disciplina que coincide con la dictadura militar (19731989). Durante este tiempo la sociología fue duramente reprimida y se eliminó su base institucional, en las universidades, clausurando o interrumpiendo carreras y centros de investigación y disminuyendo los recursos públicos que las financiaban. Todo ello afectó la formación e investigación universitaria, reduciéndolas a problemáticas muy parciales. Como contraparte, comenzaron a crearse centros académicos independientes (CAI) de carácter extra universitarios. Así, entre 1976-1980 emerge un nuevo modelo de ciencias sociales que se consolida en los 80' (GARRETÓN, 1994; BRUNNER, 1986).

A partir de 1990 - tercer período - con la recuperación del régimen democrático, comienza la reformulación de un nuevo modelo de ciencias sociales, que no satisface del todo las expectativas creadas por este proceso de democratización. En parte se modifican los contenidos de las ciencias sociales, con la reinserción de las disciplinas en las universidades y la incorporación de muchos sociólogos(as) en puestos gubernamentales que hicieron aparecer nuevas tendencias, pero manteniéndose procesos de construcción de conocimiento que permanecen segmentados, rasgo propio de la época anterior. Respecto de los CAI, con el fin de la dictadura 
y los nuevos requerimientos del Estado, la sociedad y el mercado, ese modelo fue perdiendo su vigencia, quedándose en muchos casos reducido a consultoras u ONGs más orientadas a la acción que a la investigación.

En el contexto descrito, la vocación crítica de la sociología, tanto en la etapa de fundación y consolidación como durante la dictadura, se tradujo en una función aliada de los proyectos de transformación del orden vigente. En cambio, a partir de la transición a la democracia e incluso desde mediados de los 80', no parece predominar una visión teórica crítica para el conjunto de la sociedad. La producción durante el período post-autoritario, aunque modificada en parte en los últimos años, se caracteriza por el cambio desde estudios o ensayos más globales e interpretativos sobre la sociedad, hacia estudios empíricos más monográficos y sectoriales, con un especial énfasis en las dimensiones metodológicas y técnicas tanto de la recolección de datos como de su análisis. Además, las ciencias sociales y la reflexión más general sobre la sociedad dejan de tener una teoría crítica de la sociedad en su conjunto que sea a la vez desciframiento de sus tendencias y proyección de la sociedad deseable. La sociedad parece desgajada o fragmentada en diversas esferas en que la idea de totalidad, inseparable de la dimensión intelectual crítica, parece haber desaparecido. Pero también se da lo opuesto con estudios donde esta totalidad, definida como neo-liberalismo, impide el conocimiento de micro dinámicas sociales relativamente autónomas (GARRETÓN, 2005).

En el campo profesional predomina hoy una multiplicidad de oficios y tecnologías sociales, y la unidad, siempre problemática y contradictoria, entre las dimensiones intelectual, científica y profesional que caracterizó el "ejercicio" de los cientistas sociales durante los años 60' y durante la dictadura, parece desmoronarse (GARRETÓN, 2005).

Respecto de la relación entre investigación social y la toma de decisiones a nivel de la gestión se ha señalado que ella es aún escasa y hay posicio- 
nes divergentes en cuanto a su calidad. Para unos, la información producida por las investigaciones ha sido insuficiente, pero buena y ha contribuido a tomar decisiones públicas; para otros, tal calidad ha sido mejor en las áreas físico-matemáticas que en las ciencias sociales; asimismo, la calidad ha dependido del prestigio del investigador/a, de la institución patrocinante y de la comunicación entre Estado y Academia (ANSOLEAGA, 2005), a lo que podría agregarse por nuestra parte el diagnóstico basal de la situación en que se va a desarrollar la política pública y la investigación de contexto o marco ideológico que define los objetivos de las políticas. Recordemos que para el caso chileno, la relación entre investigación y políticas públicas fue muy significativa tras el término del régimen militar, en virtud de la gran producción de conocimiento por parte de Centros de Investigación Independientes (CAI) que se habían formado bajo el régimen militar y proveyeron insumos importantes para los primeros gobiernos de la Concertación 4 . Así que por un lado, el conocimiento que se produce en las investigaciones que se realizan desde y para el Estado, en la medida que están vinculadas a las políticas públicas de democratización política y social (aumento de la equidad social y mejoramiento general de la calidad de vida de los ciudadanos), permitiría suponer un componente de crítica y compromiso social de parte de las investigaciones. Pero, por otro lado, tales políticas tienen que responder a las necesidades de eficiencia, eficacia, racionalidad, focalización y evaluación que orientan muchas de las políticas públicas en el contexto de la modernización del Esta-

4 Ejemplo significativo de esto son las investigaciones del CIDE sobre educación que fundamentaron, entre otros, el programa para las 900 escuelas básicas de mayor vulnerabilidad. En los gobiernos de Aylwin, Frei y Lagos, buena parte del equipo económico y parte del equipo social eran investigadores de Cieplan que habían escrito sobre esos temas. Posteriormente, ellos mismos fueron los tomadores de decisión y, por tanto, la influencia fue extremadamente directa. Por ejemplo, durante la reforma tributaria de los años 1990 y 1991, se pensó en los centros de estudios para la fundamentación de ésta. Por entonces había una acumulación de material sobre pobreza y distribución del ingreso y sobre cómo responder a esos problemas (ANSOLEAGA, 2005). Tales centros recibieron durante la época dictatorial un importante financiamiento internacional que fue reduciéndose significativamente en el período democrático. 
do. En todo caso no puede hablarse de intervenciones neutras, pues surgen en un proceso político y se deciden políticamente (LINDBLOM, 2000).

Que las ciencias sociales no sean neutras políticamente, no significa que sus opciones teórico-metodológicas científicas se "decidan" políticamente, porque hay tensiones entre sus dimensiones científicas, profesionales e intelectuales, y justamente esta última reivindica el compromiso normativo con nuestras sociedades y su futuro (GARRETÓN, 1994). Si bien esto no es nuevo, después de los momentos, primero, de ideologización de las ciencias sociales, luego, de persecución de las ciencias sociales bajo la dictadura militar por la vinculación previa con los proyectos políticos de transformación del país, y, finalmente, de su tendencia a la tecnocratización e instrumentalización tanto por parte del aparato estatal como del mercado, parece necesario replantearse las relaciones entre conocimiento y política.

Por lo tanto, de lo que se trata no es sólo analizar cómo las políticas públicas aplican y/o usan la investigación social, sino también abrir el debate para mostrar que tales estudios no son sólo un conocimiento experto que usan los decisores, sino que sus usos pueden fortalecer la calidad de nuestra democracia, Si bien el objetivo de toda política pública es abordar un problema público sea resolviéndolo o aminorándolo, el campo de las políticas públicas incorpora también la inacción, la carencia de políticas o las políticas implícitas y los proyectos socio-políticos de largo alcance. Ello es particularmente importante cuando se aborda este campo desde la perspectiva del uso del conocimiento (KRAFT; FURLONG, 2004). Porque, y para retomar la conjunción entre los desafíos para las dos esferas (las políticas públicas del Estado y el desarrollo disciplinario de las ciencias sociales), cabe recordar que la tradición de las ciencias sociales tiene un fuerte vínculo con las reformas sociales, por lo que la utilización de la investigación de estas disciplinas en las políticas públicas no hace sino retomar esos orígenes (HOWARD, 1788, en De YANZI FERREIRA, 2002; BOOTH, 1899). Y si 
pensamos esa relación en nuestra región, considerado el desarrollo que han tenido las ciencias sociales en Chile en particular, podemos concluir que éstas han nutrido a los proyectos políticos de país y éstos a su vez han influido en los campos disciplinarios (GARRETÓN, 2007a).

Es en este marco que nos interesaba indagar en las características y usos de las investigaciones que se hacen para el Estado. En lo que sigue presentaremos los principales resultados del análisis de los diversos estudios que se han realizado en los algunos ministerios en Chile entre 1992-2005.

\section{Quiénes investigan para el Estado: investigadores y condiciones}

En primer lugar, nos preguntamos por la formación disciplinaria de los investigadores que realizan estudios para el Estado Entre los investigadores con grado, la sociología ocupa el primer lugar en tanto está presente en el $66 \%{ }^{5}$ de los estudios, siendo más importante cuando se trata de ministerios de la política tradicional (ascendiendo al 73,3\%, a diferencia del 59\% para la nueva política y del $20 \%$ para el ministerio político gubernamental); le siguen el resto de las ciencias sociales, lo que llega a poco más del tercio (34\%), pero respecto que tienen una presencia de casi el doble en el ministerio político gubernamental $(60 \% \mathrm{v} / \mathrm{s}$ el $28,8 \%$ y $38,5 \%$ para la tradicional y nueva política respectivamente), en tercer lugar hemos reagrupado otras ciencias no sociales (26\%), que tienen mayor peso también en el ministerio político gubernamental ( $50 \%$, v/s el $27,4 \%$ y $17,9 \%$ para la tradicional y nueva política respectivamente) $y$, finalmente, las humanidades que llegan al $13 \%$ con porcentajes similares para la tradicional y nueva política.

5 Estas cifras, y las que siguen, deben ser consideradas como tendencias, en tanto nuestro proyecto FONDECYT es un estudio exploratorio, basado en un muestreo no probabilístico, y que debió realizarse en el contexto de innumerables problemas de acceso a las investigaciones. 
En el caso de los investigadores con formación de post-grado, pasa al primer lugar el postgrado en ciencias sociales sin incluir sociología (42\%, mucho más alto en el ministerio político gubernamental con un 90\%), muy de cerca queda en segundo lugar la sociología (39\% que sube bastante más en las unidades ministeriales de la nueva política con un 60\%). En tercer lugar, figuran nuevamente las otras ciencias (21\%, sólo presente - y en porcentajes similares - en la vieja y nueva política); en cuarto y último lugar están las humanidades (20\%).

En segundo lugar, sobre los enfoques disciplinarios utilizados en las investigaciones, casi un $40 \%$ son investigaciones multidisciplinarias de ciencias sociales, seguidas con un poco más de un tercio (36\%) por las que se conciben sólo desde una disciplina de las ciencias sociales (dentro de las cuales se consideraron sociología, antropología, psicología, psicología social, ciencia política y economía), mientras que cerca de un cuarto $(26 \%)$ corresponden a investigaciones multidisciplinarias que incluyen disciplinas externas a las ciencias sociales. Esta primacía de la trans o multidisciplinariedad es coherente con una de las características básicas de los problemas públicos: su interdependencia. Tal característica exige que la implementación de las políticas públicas -o paquetes de políticas- tenga altos niveles de coordinación y articulación (DUNN, 2004), es decir, se base en una integralidad de enfoques, por lo que la multidisciplinariedad interna y externa a las ciencias sociales, resulta funcional a dicha gestión. Aún más, la importancia de la multidisciplinariedad tiene relación con la definición misma de las políticas públicas y de las formas de estudiarlas y comprenderlas. Hay que recordar que el enfoque de las políticas públicas parte de una concepción multidisciplinar, en la que la Ciencia Política asume una función predominante, basada en la idea de la complejidad de la unidad de análisis y, por ende, la imposibilidad de reducir los estu- 
dios sobre políticas públicas a los parámetros de una disciplina académica o de una sola teoría (AGUILAR, 1992) .

En tercer lugar, estudiamos la externalización en la producción de conocimientos en el Estado. En nuestra muestra, en más de la mitad de los estudios (54\%) los ministerios actúan sólo como contraparte, vale decir, determinan las bases de las investigaciones y las encargan a un ejecutor externo; muy por debajo, cerca de un tercio (33\%) son ejecutadas totalmente por los ministerios; mientras que el diferencial corresponde a la situación mixta: el ministerio realiza una parte del estudio y externaliza otra. Ello no es igual en todos los ministerios, los más nuevos como SERNAM y/o más políticos como la SEGPRES, casi en su totalidad externalizan los estudios; por el contrario, sólo la DIRTRAB supera el nivel de ejecución directa con poco más del $70 \%$, lo que en los restantes ministerios sectoriales no supera el $40 \%$. Esto parece indicar que los ministerios clásicos cuentan con una mayor capacidad instalada para realizar directamente sus propios estudios, mientras que las agencias más nuevas - como SERNAM - necesitan apoyarse en circuitos expertos que están fuera del Estado. Mientras que SEGPRES, como ministerio político, puede necesitar apoyarse en sus redes de confianza para, por ejemplo, encargar encuestas sobre aprobación al gobierno.

Esta tendencia a la externalización de los servicios es coherente con el discurso que aboga porque el Estado delegue en otras instancias más especializadas parte de sus funciones tradicionales, como una forma de incrementar su efectividad y eficiencia reservándose para sí las funciones de regulación. Pero, por otro lado, lo cierto es que ello no favorece el vínculo entre la generación de políticas públicas y la producción de conocimiento científico social. Así, independientemente que muchos estudios requieran condiciones de producción académica que están mejor im-

6 Sobre el desarrollo de la perspectiva teórica de las políticas públicas ver De Kostka Fernández (2007), Aguilar, (1992), Delgado, 2002). 
plementadas en los organismos especializados externos a los ministerios (centros de estudios, universidades, consultoras, etc.), debiera fortalecerse la capacidad de los organismos ministeriales para realizar directamente parte de las investigaciones que necesitan, de modo que puedan, a partir de las mismas, generar sus propias políticas de investigación.

Respecto del tipo de ejecutores externos que realizan estudios para el Estado priman los organismos institucionalizados por sobre las personas naturales que son sólo un $12 \%$ de los ejecutores. A su vez, casi la totalidad son realizados por una sola institución, sólo el 9,5\% son realizadas por duplas de organismos. Y respecto del tipo de institución externa, en primer lugar aparecen las consultoras privadas que están presentes en el $51 \%$ de las investigaciones, seguidas de las universidades con el $24 \%$; y en tercer lugar se ubican los centros de investigación con un 12\% (categorías no excluyentes). Lo interesante es el cruce por tipo de ministerio: las universidades tienen un peso bastante mayor en los estudios encargados por las unidades ministeriales clasificadas como de la política clásica respecto de los de la nueva (51\% v/s 13\%); proporción que se invierte para el caso de las consultoras (33\% para la orientación clásica v/s 57\% para la emergente). Una posible explicación es que en la política más tradicional se privilegian las relaciones con los organismos más clásicamente encargados de la producción de estudios, ya sea por vínculos previos establecidos a lo largo del tiempo o por afinidad de los contenidos temáticos.

En cuarto lugar, más allá de estos datos que abren una serie de preocupaciones en cuanto a la relativamente baja participación de las universidades, la relación de las instituciones ejecutoras - tanto los departamentos de estudio de los ministerios como las instituciones externas a las que se encargan - con los investigadores, muestra una escasa visibilización de los investigadores en los documentos que dan cuenta de los estudios. Lo más común es que los investigadores aparezcan sólo de 
modo secundario $(40,5 \%)$ y que en un porcentaje similar $(38,5 \%)$ no haya indicación alguna respecto de quienes realizaron el estudio. En cambio, que los investigadores se ubiquen en un lugar principal del texto es poco recurrente $(16 \%)$. En todo caso, señalar a los investigadores como parte de los autores principales ocurre más a menudo cuando el ejecutor es un ministerio $(72 \%$, ) que cuando se trata de una institución ejecutora externa (62\%). En principio, para el campo académico, esto no es problema cuando las instituciones investigadoras son ampliamente conocidas y registradas académicamente. En efecto, son comunes los casos de organismos internacionales, universidades u organismos públicos que producen conocimientos fiables y muy difundidos sin indicar autores. Pero cuando se trata de consultoras no conocidas o no legitimadas académicamente, menos institucionalizadas o de existencia precaria, la invisibilización de los autores plantea una cuestión de accountability del conocimiento, lo que podría ser de importancia en ciertos casos.

En quinto lugar, respecto del tamaño de los equipos de investigación, éstos son relativamente pequeños. El promedio de investigadores por cada uno de los estudios es de 3 sujetos, con una mediana también de 3 y una baja desviación. El 26,3\% de los estudios fue realizado por un solo investigador/a, el $41 \%$ por dos o tres investigadores/as y el $32 \%$ por cuatro o más ${ }^{7}$. Los estudios realizados por un solo investigador suben del $26 \%$ al $84 \%$ cuando se trata del ministerio político gubernamental, mientras que los equipos de investigadores están presentes tanto en los ministerios de la tradicional como la nueva política, lo que podría explicarse por el énfasis menos empírico y más interpretativo del primero.

7 Con todo, se trata de tendencias, porque este dato pudo establecerse sólo para el $63 \%$ de los casos. 
Si consideramos que, además, los estudios suelen realizarse en un período bastante corto de tiempo, en general no superan los seis meses cuando mucho, cabe preguntarse si, con poco tiempo y equipos de investigación también pequeños, se cuenta con los recursos necesarios para llevar a cabo estudios de una cierta envergadura Nuestra impresión es que esto también afecta los temas y las formas en que se investiga, disminuyendo la probabilidad de realizar investigaciones sobre cuestiones estructurales, acumulativas y cambiantes en el tempo, para las cuales se requieren - por ejemplo - estudios longitudinales.

Finalmente, respecto de la composición de estos equipos por género, podemos observar que en términos generales hay más mujeres investigando que hombres (221 v/s 185). Las proporciones según sexo de los investigadores en los respectivos equipos de investigación no presentan grandes diferencias en relación a las medidas de tendencia central y de dispersión porque tanto para hombres y mujeres se mantiene la mayor concentración de casos en las categorías de uno o dos investigadores/as. La mitad de las investigaciones son realizadas por equipos mixtos (48\%), en tanto las realizadas por equipos sólo de mujeres alcanzan el segundo lugar (32\%), lo que se puede explicar por la presencia de SERNAM en la muestra, siendo más escasas las que están a cargo de equipos sólo masculinos (19\%). Lo que resulta significativo es que la presencia de equipos exclusivamente de hombres se concentra en el ministerio más político (llegando al 53,8\%), así como aquellos compuestos sólo por mujeres predominan en lo que hemos calificado como nueva política $(47,8 \%)$, A su vez, los equipos mixtos son más comunes en los servicios de la política tradicional (58\%). En cuanto a las diferencias de género, los datos muestran que los estudios realizados por un solo investigador corresponden mayoritariamente a hombres y que, al revés, las mujeres tienen más presencia en los equipos más numerosos; los equipos sin ningún hombre llegan al 32\% v/s el 19,5\% de equipos sin 
presencia de mujeres; el 36\% de los estudios donde hay presencia de investigadores hombres son equipos de dos o más investigadores, lo que sube al $46 \%$ cuando se trata de estudios que cuentan con investigadoras. Dicho de otro modo, cuando hay mujeres, los estudios son realizados por equipos más grandes respecto de los realizados por hombres.

Lo anterior deja abierta una serie de interrogantes - que aquí no desarrollaremos - en consonancia con las reflexiones de las epistemólogas feministas respecto tanto de la discriminación de género en las actividades ligadas a la ciencia ${ }^{8}$, como respecto del modo en que la dimensión de género, ya sea en cuanto postura teórica o como campo temático, cuestionan las formas de hacer ciencia androcéntrica (GUZMÁN; HOLA, 1996).

\section{Qué se investiga para el Estado: los campos temáticos}

La investigación que se hace desde y para el Estado se inscribe en lo que Gibbons, Nowotny y Scott (2003) denominan como el segundo modo de producción de conocimiento (“modo 2 ") ${ }^{9}$. Las principales características de este tipo son la transdisciplinariedad, la heterogeneidad y diversidad organizacional, el reforzamiento de la accountability, sistemas amplios de control de calidad, y además, como principal rasgo distintivo, este modo de producción de conocimiento se genera en un contexto de uso, el que describe el ambiente completo en el que surgen los problemas científicos, se desarrollan las metodologías, se difunden los resultados y se definen los tipos de usos (GIBBONS et al., 2003).

8 Existen estudios que dan cuenta de las discriminaciones de género mediante prácticas tales como que los investigadores responsables de los proyectos suelen ser hombres, que las mujeres están más vinculadas a la gestión que a la investigación en las universidades, que las publicaciones ISI en donde aparecen mujeres, suelen estar bajo el alero de un hombre y aquéllas en donde publican solas son escasas. Ver datos para una institución chilena en Araujo y Moreno (2005), para Argentina De Filippo y Serial (2003).

9 El primer modo de producción de conocimiento (modo 1) es la investigación o ciencia pura. 
El ámbito de investigaciones desde y para el Estado en la formulación de las políticas públicas, parte generalmente, del supuesto de la mayor relevancia del análisis estrictamente técnico del proceso de elaboración e implementación. Así, el conocimiento producido para la formulación de políticas públicas posee un importante anclaje territorial y una escasa movilidad de transferencia, porque es producido en un contexto de aplicación (BOISIER, 2001). Estas condiciones privilegian el método y protocolo de la investigación científica propia del paradigma empírico analítico, siendo ejemplo de aquello los análisis de costo-beneficio, el que aplicado en su forma pura desecha la influencia de otros actores y de procesos sociales y políticos (DELGADO, 2002). Esta visión anglosajona (AGUILAR, 1992), orientada fundamentalmente al problema de los medios o instrumentos, es la que ha predominado en materia de análisis, formulación e implementación de las políticas y en el énfasis en la eficiencia y la eficacia como medios para abordar un determinado problema público. Pero éste no es el único modelo de investigación existente, es decir, no hay un único modo de relación entre las políticas públicas y la investigación social. En este sentido, la contribución de la escuela europea de políticas públicas se mantiene en la tradición sociológica de abordar el análisis en la perspectiva del "por qué" y el "para qué", así como en el papel del Estado en las sociedades modernas (MULLER, 1995 citado por DELGADO, 2002). Por lo tanto, con esta segunda perspectiva, podemos abrirnos a distintos tipos de investigación para las políticas públicas, donde importen no sólo los medios sino también los fines vinculados a los problemas públicos.

En este marco, la investigación en ciencias sociales que se hace desde y para el Estado condiciona en gran medida los qué y cómo investigar, lo que lleva a preguntarse por el tipo y contenido de los estudios que se están realizando en esa instancia. 
Para conocer los temas que han investigado los ministerios incluidos en la muestra, se estableció una serie de campos temáticos de estudio ${ }^{10}$.

Los tres campos temáticos más importantes son Economía (31\%), que incluye las investigaciones que abordan temas de trabajo, mercado y política económica; seguido de Género con un $26 \%$, que incluye los temas de género, familia y sexualidad; y en tercer lugar figuran los temas de derecho con un 21\%, que reúne los temas de derechos sociales y culturales ${ }^{11}$. Respecto al campo temático de la Economía, además de la DIRTRAB, perteneciente al ámbito de la política tradicional, son SERNAM y MIDEPLAN, ambos del ámbito de lo que hemos llamado la nueva política, los ministerios que poseen los mayores porcentajes de estudios en este campo ( $37 \%$ y $33 \%$ respectivamente), mientras que en el caso de la SEGPRES este campo alcanza una presencia nada de despreciable con la quinta parte de sus investigaciones. Por su parte, el campo temático de Género, asociado a la nueva política, si bien es dominante la presencia de SERNAM, en MIDEPLAN alcanza un $23 \%$ de las investigaciones que realiza, mientras que en los ministerios de la política tradicional no alcanza al $7 \%$ de sus investigaciones. En cuanto a las temáticas relacionadas con Derechos sociales y culturales, fuera de MINEDUC, ellas se concentran en MIDEPLAN, SERNAM y la DOS pertenecen a este campo. En otras palabras, en relación a estos tres campos temáticos, ellos se encuentran fuertemente asociados a los ministerios escogidos en la muestra.

10 Recordemos que cada campo está condicionado por los ministerios considerados. Por ejemplo, dado que incluimos a SERNAM que trabaja los temas de género, y el peso muestral de sus investigaciones es del 14\% -como el de cada uno de los restantes ministerios- lo más probable es que al indagar las áreas en las que se investiga en el Estado, el tema género a lo menos bordee ese porcentaje.

11 Dentro del campo temático de derechos, se excluyó la temática de género, ya que éste es un tipo de derecho transversal a otros derechos. 
En relación al conjunto de campos temáticos, tres otros datos parecen importantes de considerar. Por un lado, se aprecia una fuerte focalización de algunos ministerios en algunos campos, como MINVU en el campo de la Sociología Urbana y la DOS en el de la Participación Social, tema este último de baja presencia en los ministerios de política tradicional. Por otro lado, en los temas de Evaluación, Metodología e Intervención, son MINEDUC y MIDEPLAN los ministerios con mayor representación en este campo, en donde ambos tienen un tercio de sus investigaciones, lo que puede tomarse como un indicio del uso que se les da en el Estado a las investigaciones en ciencias sociales En tercer lugar, la Gestión del Estado y las Políticas Públicas se encuentran dentro de los campos poco estudiados, lo que significa que el Estado suele no estudiarse a sí mismo ni su forma de actuar, como tampoco, en la época de sociedad de la información se cuenta con una mayor presencia de investigaciones referidas a estos temas (por ejemplo, exclusión de la información y/o exclusión digital). Al menos en los ministerios estudiados, el Estado muestra ciertos déficits de investigaciones en los nuevos temas que expanden la responsabilidad gubernamental, sobrepasando los temas clásicos de la administración pública como salud, educación, servicios sociales y desarrollo económico entre otros (BORINS, 2008).

Un matiz importante que se observa entre los Ministerios de la política tradicional y los de la nueva política, es que en los primeros los temas que escapan a su ámbito tienen una importancia considerablemente menor, mientras que en los segundos se cuenta con al menos tres temáticas centrales, pero acompañadas de otra serie de temáticas con presencia nada de despreciables (como temas de Estratificación, Política y Evaluación, Metodología e Intervención por nombrar algunos), lo que nos indicaría una mayor transversalidad temática en la nueva política en contraste a la especificidad y focalización de los temas de la política tradicional. Es probable que esta transversalidad de la nueva política obedezca a una necesidad de validación social y estatal de estos temas. 
Mirando los campos temáticos en una perspectiva temporal, distintos autores coinciden en que durante la década del 80' la investigación social desarrollada desde y para el Estado en Chile, se preocupó principalmente de la especificación y aplicación del modelo neoliberal, lo que junto al control militar de las universidades, aseguró tanto la enseñanza como la investigación a partir de la nueva verdad de la economía (GARRETÓN, 2007a, p. 212). No obstante, a partir de la recuperación de la democracia, podemos destacar el impulso que vive la Ciencia Política como disciplina y que se refleja tanto en los campos temáticos como en los sujetos focos de estudio (actores del mundo político civil y los actores del mundo gubernamental). No obstante, a pesar del aumento de las investigaciones sobre la profundización democrática que caracterizó al período 1990-1997 (GARRETÓN, 2007a, p. 230), el predominio de los temas de economía y el énfasis en los actores del mundo económico (trabajadores, empresarios, microempresarios, empresas y sindicatos) es manifiesto. Ello significaría que en vez de darse una sustitución del foco temático principal, se da una diversificación en los campos temáticos, adquiriendo mayor importancia los temas políticos y los temas de derechos (sociales y culturales). Ello, a su vez, rompe con una visión esencialista del Estado, de la nación y sus instituciones, en provecho de sus manifestaciones socio-históricas siempre en movimiento y en disputa (IHL; KALUSZYNSKI, 2002), las que se traducen en nuevos focos de interés y radios de acción para el Estado. Esta ampliación de los temas que investiga el Estado, nos recuerda que la intervención planificada del Estado a partir del siglo XIX en distintas áreas, se justificaba en un principio en las ciencias que las amparaban y así se maximizaba la efectividad del trabajo burocrático (IHL; KALUSZYNSKI, 2002) ${ }^{12}$. En los siglos XX y XXI se añadirían a las ciencias

12 Un buen ejemplo de cómo las ciencias permiten el incremento del accionar del Estado, lo encontramos en la El nacimiento de la clínica (1963) de Michel Foucault, para el caso de la medicina especialmente. 
naturales, las ciencias sociales como las nuevas "ciencias útiles" que permiten al Estado ampliar sus ámbitos de acción.

En parte puede ilustrarse esta ampliación de los campos de acción del Estado y la búsqueda de conocimiento para ello, con el surgimiento de estudios sobre exclusión, reflejando la importancia que adquieren como sujetos foco de estudio los grupos vulnerables y/o discriminados (niños, jóvenes, mujeres, migrantes, ancianos, presidiarios, desempleados) que alcanzan al $45,5 \%$ de las investigaciones estudiadas, situándose sólo por detrás de los actores del mundo económico (47,6\%). En esta línea, un tercer grupo de actores estudiados por el Estado, está compuesto por aquellos definidos por la posición que ocupan en la estructura social (como los pobres urbanos, los pobres rurales o las capas medias), siendo MIDEPLAN y MINVU los ministerios que concentran los mayores porcentajes de investigaciones centradas en estos actores. Asimismo, en esta parcelación y especificidad en la producción de conocimientos, destacan los estudios sobre temáticas culturales que han ido adquiriendo mayor importancia entre de las investigaciones hechas a partir del Estado desde la segunda mitad de los 90' hasta la fecha, como se expresa en la importancia que adquieren los temas de Género (GARRETÓN, 2007a).

\section{Cómo se investiga para el Estado: enfoques y metodologías}

Para entender el uso que se le da a las investigaciones en ciencias sociales realizadas desde y para el Estado, es relevante conocer las características metodológicas de los estudios, vale decir, conocer el cómo se investiga.

La caracterización de un problema, sus factores asociados e implicancias sobre otros, nace de la observación y la aplicación de unos instrumentos metodológicos que serían proporcionados por determinadas disciplinas, dependiendo de la orientación o política más global, que es- 
tablecería cual dimensión del problema es la que se quiere abordar y qué actores estarían comprometidos. En este sentido, si bien no todos los investigadores tienen algún interés práctico (AURIAT, 1997) la investigación en ciencias sociales puede ser un instrumento útil para perfeccionar el proceso de elaboración de las políticas públicas, en la medida que la información que se recabe ocupe un lugar en el proceso de toma de decisión en consonancia con la orientación de los actores intervinientes.

Al respecto, podemos señalar que las investigaciones realizadas por el Estado, si bien caben en lo que se ha llamado segundo modo de producción de conocimiento, en general cumplen con los parámetros de lo que se considera proyectos y reportes de investigación en el ámbito académico o del primer modo de producción de conocimiento de la "ciencia pura" (GIBBONS et al., 2003). Así, casi la totalidad (92\%) de los informes de investigaciones analizados indican de alguna forma un problema de investigación, un objetivo general o alguna descripción de lo que se pretende desarrollar. No obstante, este nivel baja a poco más de la mitad (56,7\%) cuando se trata de la inclusión explícita de objetivos específicos, que son los permiten acotar la manera en que el problema de investigación será trabajado. Por otra parte, en la gran mayoría de las investigaciones (80\%) sus resultados dan cuenta de lo que los objetivos pretendían, y en un $85,1 \%$ de los reportes se estableció la presencia de conclusiones explícitas.

En cambio, la manera en que se expresa la teoría en estas investigaciones es distinta a la forma en que opera en los estudios más propiamente académicos, lo que se explica en parte porque a diferencia de las investigaciones realizadas en el marco del primer modo de producción de conocimiento más relacionado con la ciencia básica, en los estudios realizados desde o para el Estado el objetivo no siempre se refiere productos convencionales como las publicaciones académicas en donde las teorías y conceptos ocupan un lugar más importante (GIBBONS et al., 
2003). Así, al preguntarnos por la presencia de teorías, enfoques, autores o conceptos dentro de las investigaciones, lo más común es que haya un capítulo o apartado específico para el marco teórico, pero ello cubre sólo a cerca de un tercio de los estudios $(34,3 \%)$, mientras las referencias teóricas en la contextualización o presentación del estudio bordean el $20 \%$ y en más de una cuarta parte de las investigaciones (27\%) no hay presencia de teorías, enfoques o conceptos. El desarrollo de antecedentes teóricos en un capítulo específico es más frecuente en el ministerio político gubernamental; mientras que las referencias teóricas en la contextualización o en la presentación de las investigaciones son levemente más frecuentes en los ministerios de la nueva política; y los estudios en los que no hay presencia de antecedentes teóricos se dan más en los ministerios de la política tradicional y nueva que en el ministerio político gubernamental.

Con esto, aunque sin total nitidez, pareciera consolidarse un tipo de investigación que no se basaría en antecedentes teóricos para su desarrollo, lo que hubiera sido impensable hace un par de décadas en el mundo de la investigación en ciencias sociales. Es probable que este cambio obedezca a que en el segundo modo de producción de conocimientos hay una mayor referencia a los usos de la investigación y al diseño, seguimiento y evaluación de planes, programas y políticas desarrolladas a partir del Estado y menos referencia a una acumulación y debate y teóricos. Ello, al menos para la sociología, es un desafío no menor porque más allá del instrumental metodológico, la dimensión heurística, crítica, de comprensión y aporte a la transformación de los fenómenos sociales en las sociedades históricas en las que nos desenvolvemos, difícilmente pueden darse sin teoría. Por lo demás, los enfoques teóricos son no sólo herramientas analíticas, sino también campos de disputa ideológica y están presentes aún cuando no se expliciten, de ahí el desafío de encontrar nuevas formas para vincularlos a la investigación para las políticas 
públicas, de modo de no invisibilizar los enfoques que participan en las disputas sobre lo que es o no un problema público y cómo abordarlo y desde los cuales se construye el conocimiento.

Aún así, la mayoría de los estudios (74\%) presentaba un desarrollo teórico que a nuestro juicio parecía ser suficiente para entender la perspectiva desde la que se desarrollaba la investigación. En cuanto al uso de la teoría o enfoque para interpretar los datos de cada investigación, ello también ocurría en la mayor parte de los estudios (70\%), mientras que en un $22 \%$ de las investigaciones se mencionaban autores o enfoques, pero luego ellos no se utilizaban en la interpretación de los datos.

Para saber si las investigaciones estudiadas hacían uso de información de tipo secundaria, nos preguntamos si es que en ellas existía diálogo o referencia con otras investigaciones. El 78,1\% de las investigaciones que componen esta muestra hacen referencia o dialogan con otras investigaciones y en aproximadamente la mitad de ellas se hacen referencias a investigaciones del mismo Ministerio, del Estado, a otros estudios de la disciplina y a bases de datos. A los estudios a los que se hacen menos referencias son los a de autor, a los de las mismas instituciones ejecutoras externas, y a estudios de otras disciplinas (con porcentajes del 23,8\%, 13,4\% y 16,5\% respectivamente). Puede especularse que tanto las instituciones como los investigadores mayormente no desarrollan líneas de especialización en determinadas temáticas, y que ese podría ser el motivo por el cual no hacen referencias a anteriores trabajos en la materia, debido a que parecen no contar con los antecedentes al respecto. De nuevo ello tiene importantes consecuencias en la acumulación (réplicas, avances, refutaciones y rectificaciones, nuevas líneas en la misma temática, etc) del conocimiento por parte de las entidades encargadas de las políticas públicas.

Una de las características más importantes del segundo modo de producción de conocimiento, como se ha indicado más arriba, es la multi 
o transdisciplinariedad, la que puede ser vista en el uso de diversas metodologías, pero sin que necesariamente se llegue al refinamiento de éstas o a la creación de otras nuevas (GIBBONS et al., 2003), por lo que examinar los marcos metodológicos de las investigaciones es clave para dar cuenta de esta dimensión, para lo que contamos con varios indicadores.

En primer lugar, sólo en la mitad de los casos (55\%) se presentan referencias metodológicas de manera clara, casi un tercio (32\%) indica algunas referencias, pero tales resultan insuficientes si se quiere conocer cómo se realizó la investigación, mientras que en un 13\% no hay referencias metodológicas. A su vez, las investigaciones con más referencias metodológicas son características de los ministerios de la política tradicional, mientras que los de la nueva tienen una mayor proporción de estudios con referencias insuficientes; y la ausencia de antecedentes metodológicos prevalece en los estudios del ministerio político gubernamental, lo que puede deberse a que en este caso hay más estudios que prescinden del trabajo de terreno. Por otro lado, la mayoría de las investigaciones utilizan diseños de tipo no experimental, y los pocos casos de estudios experimentales o cuasi experimentales se vinculan a la política tradicional, lo cual hace suponer que los niveles de complejidad de este tipo de investigaciones se corresponden con el grado de consolidación de las temáticas de estudio de tales ministerios o bien de los mismos ministerios.

Respecto a los tipos de estrategias metodológicas seguidas por estas investigaciones, tenemos que en un $42,2 \%$ de los casos éstas siguen una estrategia de tipo cuantitativa (exclusiva o principalmente), mientras que el 32\% utilizan la cualitativa (exclusiva o principalmente). El resto corresponde a diseños mixtos. En cuanto a su distribución según los tipos de ministerios, en la política tradicional la mitad de sus investigaciones siguen una estrategia cuantitativa a diferencia de la nueva política donde es poco más de un tercio (36\%). La estrategia cualitativa domina en la nueva 
política (42\%) a diferencia de política tradicional (21\%). Los diseños mixtos no marcan diferencias. El mayor uso de estrategias cualitativas en la nueva política adquiere sentido si asumimos que sus temáticas responden a nuevos fenómenos y ámbitos donde las técnicas cualitativas son más pertinentes y suelen ser más recomendadas, precisamente porque se han desarrollado en conjunto con estos fenómenos y ámbitos.

En lo concerniente al tipo de análisis empleado en las investigaciones, la mayoría de la muestra (76\%) usa algún tipo de análisis cuantitativo, de las cuales gran parte hace uso de un tipo de análisis estadístico que se queda en lo descriptivo y/o inferencial, mientras que sólo el 7,4\% de las investigaciones que utilizan análisis estadísticos hacen análisis de tipo multivariado, lo que en el caso del MINEDUC, corresponde al 26\% de estas investigaciones. Por otra parte, los análisis de tipo cualitativos son utilizados por el $57 \%$ del total de las investigaciones analizadas, apreciándose diferencias importantes en los niveles alcanzados por los ministerios. De este grupo de investigaciones, el $80 \%$ de ellas hacen uso de las formas menos complejas de análisis cualitativo (de contenido, grounded theory y estructural entre otros), mientras que el resto de estas investigaciones utilizó el análisis cualitativo de discurso (como forma más compleja de análisis), resaltando que en más del $70 \%$ de las investigaciones de la DIRTRAB y en cerca de la mitad de las de MIDEPLAN (44\%) que utilizan algún tipo de análisis cualitativo, se hace uso del análisis de discurso ${ }^{13}$.

Si reagrupamos los tipos de análisis de acuerdo a su complejidad o grado de sofisticación ${ }^{14}$, sin que importe si son de tipo cualitativo o cuantitativo (para trabajar con la totalidad de las investigaciones que utilizan algún tipo de análisis), tenemos que sólo el 18\% de los estudios utilizan

13 Los datos de los análisis cualitativos y cuantitativos son coincidentes con los de Ramos (2005). 14 Otorgando el estatus de mayor complejidad al análisis multivariado y al análisis de discurso, para lo cuantitativo y lo cualitativo respectivamente. 
los análisis de mayor complejidad, siendo los ministerios de la política tradicional aquéllos de mayor presencia relativa en esta categoría (28\%) en comparación con los ministerios de la nueva política y el ministerio político gubernamental, cuyas presencias en esta categoría sólo alcanza el $11 \%$ y el $7 \%$ respectivamente de sus investigaciones.

En cuanto al alcance de las investigaciones, encontramos como tendencia predominante el nivel descriptivo en la mitad de los casos (51\%), sin que existan diferencias entre los tipos de ministerios. Por su parte, las investigaciones evaluativas (ex ante, ex dure y ex post) constituyen el $22,4 \%$ de los casos, lo cual si bien las ubica en un segundo lugar, muestra que aún es menor el uso de las investigaciones por parte del Estado para conocer de qué manera se implementan algunos programas y políticas en sus distintas fases. Sobre esto último, es preciso mencionar que los ministerios y servicios tanto de la política nueva como de la tradicional, tienen comparativamente mayor proporción de estudios evaluativos que el ministerio político gubernamental.

En síntesis, si bien observamos que estas investigaciones - como parte del conocimiento producido desde y para el Estado - no muestran grandes distancias con el modo 1 de producción de conocimiento en los aspectos formales de la formulación de las investigaciones, sí encontramos importantes diferencias en el uso de las teorías, los niveles de análisis alcanzados y otros aspectos metodológicos.

\section{Para qué se investiga en el Estado: acercándonos al uso de las investigaciones}

Como señalábamos al inicio, nuestro interés no es sólo analizar cómo en las políticas públicas se aplica y/o usa la investigación social, sino también abrir el debate para mostrar que tales estudios no son sólo un 
conocimiento experto que usan los decisores, sino que sus usos pueden fortalecer la calidad de nuestra democracia, en el triple sentido de mejorar la calidad de las políticas de gobierno y la respuesta a las demandas sociales, fortalecer la capacidad institucional de intervención del Estado y contribuir a la calidad del debate público en torno a los problemas sociales que deben ser objeto de intervención publica. Nuestro estudio cuantitativo no nos permite sino una aproximación indirecta al tema del uso de las investigaciones a través de tres dimensiones: la selección de los temas, el público a que llegan y la aplicación a las políticas públicas.

En lo que se refiere a la selección de temas, la cuestión central es cómo los problemas públicos son convertidos en problemas de investigación, lo que puede pesquisarse a través de la justificación de los estudios, los temas que se investigan, la forma en que se narra la vinculación con las políticas públicas. Para mencionar sólo uno de estos aspectos, digamos que entre los motivos de estudio de un determinado tema, se destaca el que éste responde a las líneas programáticas del ministerio (74\% de las investigaciones), básicamente en los de las políticas tradicional y nueva. En segundo lugar, bastante más alejado, figura que el tema responda a las líneas programáticas del gobierno y al deseo de generar una política pública relacionada con el ámbito de estudio (cerca del 30\% cada uno), criterios que son más importantes en el ministerio político gubernamental.

La segunda dimensión se refiere a cómo los estudios salen desde el ámbito de gestión de las políticas públicas al resto de la sociedad. De ahí que nos preguntamos qué tipo de lector suponían los informes y publicaciones de investigación, como una forma de acercarnos a los circuitos de influencia de estos estudios ${ }^{15}$. Así, en casi la totalidad de los estudios (91\%) el lector correspondía a un público experto en el área de las políticas

15 Se trata de categorías no excluyentes. 
públicas; le sigue casi la mitad de los estudios orientados a un lector de tipo más académico; y de lejos con casi un cuarto (23\%) un público más vinculado a los partidos políticos (dirigentes, militantes, congresistas) y sólo el 7\% suponía un lector no experto. Aún cuando el principal tipo de lector parece el adecuado si los estudios se hacen para los agentes ministeriales, podemos pensar otros usos posibles, precisamente porque la elaboración de políticas públicas es más compleja en los sistemas democráticos en tanto hay más actores que intervienen y que buscan influir en los tomadores de decisiones políticas (LINDBLOM, 1991). A la luz de estos datos, un desafío para mejorar la calidad de la democracia es potenciar la circulación de este tipo de conocimiento experto producido por las ciencias sociales con relación a las políticas públicas, de manera de ampliar el rango de quienes podrían participar, disputar o aportar en la definición de lo que consideramos problemas públicos, lo que implica que los informes de los estudios a lo menos consideren como lector potencial al mundo vinculado a los partidos políticos y al Parlamento de manera más intencionada, toda vez que ellos participan en la definición de la agenda de los problemas públicos, y, aunque es más desafiante todavía, tal circulación de conocimientos puede pensarse también para un público no experto (Ej. mediante publicaciones de difusión, charlas, capacitaciones a dirigentes locales).

La tercera dimensión se refiere a la aplicación del conocimiento a las políticas públicas. Considerando los modelos de Weiss de aplicación de la investigación en las políticas públicas ${ }^{16}$ intentamos clasificar las investigaciones en alguno(s) de ellos. Según eso, obtuvimos que el más recurrente (poco más del $50 \%$ ) es el modelo de la búsqueda de conocimientos, donde el conocimiento científico pretende servir de base a la toma de decisiones (salvo para el ministerio político gubernamental donde baja a casi un tercio). Le siguen

16 Ver Weiss, 1986, citado por Auriat, 1997. Para Auriat, sin embargo, ninguno de estos modelos responden a la pregunta por cómo operan los responsables de la toma de decisiones frente a las conclusiones de las investigaciones y su uso en la formulación de políticas. 
los modelos denominados como solución de problemas y como clarificación, cada uno con cerca de un tercio. El primero implicaría un cierto consenso entre los planificadores de políticas y los investigadores sobre los resultados y la certeza de que la investigación contribuye a definir los medios adecuados para formular la política; este modelo es más importante precisamente en el caso del ministerio político gubernamental (43\%). Mientras que el modelo de la clarificación, se basaría en la potencialidad de utilizar la investigación social para categorizar o delimitar conceptualmente las dimensiones de una política, y en ocasiones puede funcionar como catalizador de su redefinición. Según Weiss, este modelo no siempre estaría en consonancia con la orientación de los tomadores de decisión. En la muestra, sorprende que pesa más en el ministerio político gubernamental. El lugar siguiente lo ocupa el modelo táctico (13\%) correspondiente a la aplicación de una investigación impulsada desde organismos de gobierno como respuesta táctica a la opinión pública ${ }^{17}$.

Más allá de los datos cuantitativos, las dificultades encontradas en nuestra investigación para reconstruir el universo de investigaciones realizadas desde y para el Estado en los ministerios seleccionados nos permiten algunas consideraciones sobre una cuarta dimensión de aproximación indirecta al uso de las investigaciones por parte del Estado cual es la política de archivos y registro, base para una política de investigación propiamente tal.

Lo primero es que, salvo intentos aislados (Ej. MINVU y DIRTRAB), no hay una política de archivo, registro y disponibilidad de las investi-

17 Los restantes modelos, que en nuestro proyecto presentan porcentajes marginales, corresponden a lo que Weiss denomina como el interactivo y el político. A diferencia de los anteriores, el modelo interactivo plantea la interrelación tanto de diversos marcos teóricos como de actores (administradores, técnicos, políticos, planificadores, periodistas, clientes, grupos de interés, colaboradores y amigos); y en este caso, los cientistas sociales no siempre tienen un lugar privilegiado. Por otro lado, en este modelo la política suele impulsarse aún cuando no se disponga de los resultados, siguiendo a las presiones políticas en curso. El modelo político, por su parte, constituye una aplicación que bien puede ser vista como un instrumento neutralizador de presiones, para ganar adeptos. En este caso la investigación cuenta con agentes dispuestos a defenderla aumentando las probabilidades de que tenga injerencia en una determinada política. 
gaciones que ordene, procese y gestione el acceso, uso y difusión de la información y resultados. En algunos ministerios esto está empezando a armarse, en otros se venía configurando, pero se ha frenado, y en otros no existe. Ello parece tener relación con la carencia de personal encargado de la gestión de este tipo de información, ya que no hay una estructura adecuada que procese la demanda por los estudios. En segundo lugar, y aunque parezca inverosímil, hay Ministerios que no conservan o no cuentan con la mayor parte de los estudios que han realizado. En varios casos éstos se perdieron, en otros están "guardados" en alguna parte o quedaron en los sistemas de archivo de ex funcionarios; por lo mismo no siempre hay registro sobre su ubicación. En tercer término, lo anterior parece un indicio de que en general, no hay una política de investigación, ligada al diseño y monitoreo de las políticas públicas, que permita una evaluación coherente a lo largo del tiempo. La decisión de encargar o generar un estudio parece responder al estilo de gestión o al simple voluntarismo de quienes están temporalmente a cargo de las unidades responsables de generar tales estudios; a demandas coyunturales o a objetivos de gestión muy específicos. En los Ministerios donde los Departamentos de Estudio se encuentran consolidados, ha sido posible insinuar una política de investigación más clara o al menos hacer un esfuerzo de sistematización, pero en general, no se aprecia una política de archivo sistemática, ni de traspaso de información luego de la rotación de cargos, por lo tanto es difícil pensar que se pueda aprovechar esta información. Ello tiene como posibles efectos la duplicación de investigaciones, pero sin dimensión comparativa sino como simple repetición de algo que se desconoce que ya se hizo, la falta de acumulación de conocimiento y el dispendio de recursos. De aquí el desafío de pensar y elaborar una política de investigación en los ministerios, que a su vez incluya una política de archivos de la información. 


\section{Conclusiones}

Los datos que aquí hemos presentado nos permiten señalar dos grandes conclusiones que tienen que ver con los desafíos para las políticas públicas por un lado, y los retos para el desarrollo disciplinario de las ciencias sociales por el otro.

1. Teniendo en mente los resultados expuestos, necesitamos volver al contexto más amplio sobre el tipo de sociedad post-industrial globalizada en que vivimos (GARRETÓN, 2000), considerando la importancia que en ella adquiere el principio del conocimiento y de la información (CASTELLS, 1997); donde se transforman los modelos de desarrollo y de modernidad que conocimos, y el consecuente rol del Estado. En este contexto, hay que considerar que allí donde el Estado tenía un rol activo y preponderante, hoy se ve sujeto a la confrontación de intereses del ámbito privado y los que trascienden el ámbito nacional, con lo cual también cambian las formas en que se constituía una política pública. Cuestiones tales como el medio ambiente, la energía, la innovación en ciencia y tecnología, la eliminación de la pobreza, etc. implican la expansión de políticas públicas transversales. Nada de ello puede ser posible si no se toma en serio la cuestión de la relación entre Estado y sociedad que requiere hacer a éste impermeable a las presiones de los grupos de poder, económicos, comunicacionales, corporativos, que intentan identificar al Estado con sus propios intereses y, por otro lado, incorporar a la elaboración de políticas públicas la participación ciudadana tanto en funciones deliberativas, fiscalizadoras y, en ciertos casos, decisorias (GARRETÓN, 2007a). En caso contrario estaríamos avalando la tesis de la existencia de una involución en el ejercicio de la política, proceso que sería reemplazado por una seudopolítica (MOULIÁN, 2004). En los actuales niveles de conocimiento exigidos para la toma de deci- 
siones y pensando en el efecto largo plazo de las políticas públicas, se necesita un Estado que participe de las redes de producción de conocimiento como parte de la reflexividad institucional (GIDDENS, 1997).

Si lo anterior es así, la relación entre las políticas públicas y conocimiento científico social plantea la cuestión de cuáles son roles del conocimiento en y para la política, las políticas públicas en particular, pero la política como construcción de la buena sociedad en general, de modo que el conocimiento experto no invisibilice ni reemplace el debate sobre los horizontes normativos. El conocimiento tiene dimensiones políticas, y la política tiene una dimensión sobre qué, cómo y para qué conocer. Eso nos devuelve a varias preguntas relacionadas con la calidad de nuestra democracia: qué tipo de conocimiento necesitamos, cuál será su rol en la definición de las políticas que nos damos y quiénes -y cómo- participan en la producción y uso del conocimiento científico social que nos constituye. Todo ello supone contenidos, ideas, debates y acuerdos políticos. Necesitamos conocimiento experto para mejorar la generación y gestión de las políticas públicas, pero también necesitamos una democracia de calidad que permita seguir apelando al legítimo y necesario aporte de la política en la definición de los problemas colectivos y de la forma de enfrentarlos a través de las políticas públicas.

El debate público es el espacio en que conocimiento experto, ciudadanía y decidores se encuentran, de modo de evitar su pura tecnocratización y mediatización. Pero ello supone la generación de una nueva institucionalidad estatal que permita el diálogo con el sistema de producción de conocimientos. Es en función de esto que nos parece rele-

$18 \mathrm{Si}$ consideramos los resultados obtenidos en nuestro estudio cualitativo correspondiente a la segunda etapa de nuestra investigación, con entrevistas a funcionarios ministeriales encargados de investigaciones y a investigadores externos, existe la sensación más o menos generalizada entre los investigadores/as que el Estado carece de una política de investigación claramente definida, sumado al reconocimiento por parte de los funcionarios/as ministeriales vinculados a las investigaciones del Estado, de que éstas muchas veces obedecen a las contingencias en las 
vante considerar lo que antes señalamos sobre la falta de una política de investigación en el Estado chileno, orientada a la formulación, gestión y evaluación de las políticas públicas. ${ }^{18}$ Una hipótesis que podría explicar la ausencia de políticas de investigación claramente definidas, es que la investigación como tal carece en cierta medida de valor público, y ello explicaría en parte la falta de planificación que podría plasmarse en una política de investigación. Si éste fuera parte del problema, la creación de valor público a la investigación requiere el establecimiento de objetivos y metas claros, lo que exige una planificación independiente a los acontecimientos coyunturales que también requieran de estudios, es decir, una institucionalidad adecuada que incluye un sistema de registro, archivo y acceso a la información más eficiente, pero también más abierto a la ciudadanía. Se trata del fortalecimiento de la capacidad de las unidades del Estado para producir directamente el conocimiento que requieren, así como ampliar su base de investigadores externos a los que encarga los estudios. En esto parece preocupante que la mayor parte de las investigaciones que el Estado encarga para apoyar el diseño y gestión de sus políticas públicas provenga de las empresas consultoras más que de las universidades y centros de investigación.

Asimismo, es en función de ese debate público en torno a las relaciones entre conocimiento y política que se deben buscar nuevas formas de difundir los conocimientos que las ciencias sociales producen para el Estado, en tanto ellos permiten ampliar los márgenes de lo que colectivamente definamos con problemas públicos y las formas de abordarlos.

2. Por otro lado, si consideramos los datos que hemos presentado desde la pregunta por los desafíos que supone para el desarrollo de las

que se ven envueltas las unidades ministeriales, y que no necesariamente se inscriben en una política de investigación de larga data, aunque sí se mantienen líneas temáticas de investigación, lo que algunos funcionarios/as ministeriales señalan como "la" política de investigación. 
ciencias sociales, particularmente para la sociología, caben tres consideraciones principales.

Por un lado, las investigaciones que se realizan para el Estado parecen estar bien formuladas, pero son débiles en su capacidad para dar cuenta de sus enfoques teóricos y metodológicos, y a la vez deben realizarse en condiciones bastante precarias en cuanto a recursos humanos (equipos reducidos) y de tiempo (investigaciones cortas). Ello permitiría concluir que sus resultados pueden ser fácilmente convertidos en un argumento más que en un conocimiento que nutra las políticas públicas.

Por otro lado, este modo de producción de conocimientos exige la multi o transdisciplinariedad, por cuanto implica ante todo la capacidad de movilizar y manejar distintas perspectivas y metodologías capaces de generar argumentaciones. Ello porque en el "modo 2" el conocimiento es incorporado principalmente a través de la maestría de investigadores/as y equipos, antes que a través de productos de investigación convencionales como nuevas teorías, conceptos o metodologías a presentarse en publicaciones de carácter científico (GIBBONS et al., 2003). Esto se vuelve clave al observar que lo que buscan los funcionarios/as ministeriales al encargar los estudios que el aparato estatal requiere para la legitimación de las decisiones es el "expertizaje" como conjunto de competencias profesionales.

En tercer lugar, los temas que se investigan parecen no desligarse de lo que ya está naturalizado como problemas sociales (cuestiones de equidad y acceso a derechos sociales de los grupos menos favorecidos), dejando de lado temas que mejoran la gestión de las políticas públicas (dada la escasa investigación en temas de evaluación, metodologías, articulación interministerial y el propio aparato público). Ello permite plantearse hasta qué punto la investigación que demanda el Estado puede incidir en la innovación y modernización del aparato público. Asimismo, los temas y focos de investigación parecen limitarse a los sujetos de políticas sociales, 
pero no aparecen temas vinculados a la desigualdad social referida tanto a los temas clásicos de estratificación, como a los que supone la sociedad del conocimiento, y la desigualdad político-territorial. Ello explicaría la escasa investigación en temas de estructura de clases, acceso al conocimiento y temas del mundo rural.

La reflexión final es que tanto desde el lado estatal, que provee el financiamiento condicionado de las investigaciones que requiere, como del lado de la producción académica interesada en el desarrollo disciplinario principalmente, cabe realizar una profunda revisión de lo que es relevante y no relevante de investigar en sociología. Y esto puede afectar a su vez tanto la formación de las nuevas generaciones de sociólogos que demandan competencias para insertarse en el mercado laboral, orientado a la consultoría para el mundo público y privado, como las formas de pensar y enseñar nuestras prácticas de investigación. Una vez más, los proyectos académicos de la disciplina no son inmunes a los cambios en las sociedades en los que se desenvuelven y a las demandas de conocimiento de los agentes que las requieren.

De lo que se trata es de "fortalecer y rediseñar la misión y los mecanismos de producción de un conocimiento útil, pertinente y riguroso, a la vez que democrático y socialmente incluyente, utilizable (...) en términos de políticas públicas" (CARRIZO, 2004, p. 6). Ello implica transformaciones que apuntarían a cuatro ámbitos. Primero, la formación de los investigadores, promoviendo una perspectiva y una actitud transdisciplinaria. Segundo, la cultura política, fortaleciendo el diálogo entre la investigación y la política. Tercero, la cultura ciudadana, lo que supone la promoción de la participación ciudadana en la construcción de las políticas (CARRIZO, 2004, p. 6-10). Cuarto: la revisión y reformulación de la institucionalidad tanto de cada ministerio como del conjunto del aparato estatal en lo que 
se refiere a la planificación, coordinación, ejecución y acumulación del conocimiento producido por las investigaciones.

\title{
Social Science and Public Policy in Chile: what does the state research, how is it done, and what is it for?
}

\begin{abstract}
This article presents some of the results of a study on the links between social science research and the formulation and management of the public policies in Chile after the democratic transition. Through an analysis of studies conducted in traditional, new and political ministries, the article examines four issues: a) the state's researchers and their work conditions, b) the content of the research, c) methodological perspectives, and d) the use of the research. The answers to these questions provide some conclusions on two aspects: to the state, what is the impact of these studies on the quality of the public policies within the context of the debates on the quality of democracy in Chile; and to the social sciences, how does the presence of the state as one of the main applicants of the scientific knowledge affects the development of disciplines, especially the sociology.

Keywords: Research. Public policy. Sociology. Expert knowledge. State. Knowledge use.
\end{abstract}

\section{Referencias}

AGUILAR, V. Estudio introductorio, Hechura de las políticas. México, Grupo MAP, 1992.

ANSOLEAGA, María Elisa. Investigación y políticas públicas, una necesidad urgente para Chile: el fondo para el estudio de las políticas públicas como instrumento de gestión de proyectos, en Estudio de Caso n. 84, Magíster en Gestión y Políticas Públicas, Dpto. de Ingeniería Industrial, Universidad de Chile, Agosto, Santiago, 2005.

AURIAT, Nadia. Las políticas sociales y la investigación social: reapertura del 
debate, en http://www.unesco.org/issj/rics156/auriatspa.html, 1997.

BOISIER, S. Sociedad del conocimiento, conocimiento social y gestión territorial, 2001. Disponible en: http://www.idr.es/publicaciones/desarrollo.

BORINS, Sandford (Ed.). Innovations in government. Research, recognition, and replication. Washington D.C.: Brookings Institution Press, 2008.

BRUNNER, José Joaquín. Las Ciencias sociales en Chile: Institución política y mercado en el caso de la sociología. Santiago, Chile, FLACSO (Documento de trabajo n. 35), 1986.

BRUNNER, José Joaquín; SÚNKEL, Guillermo. Conocimiento, Sociedad y Política. Santiago: Libros FLACSO, 1993.

CARRIZO, Luis. Producción de Conocimiento y Políticas Públicas. Desafíos de la Universidad para la Gobernanza Democrática. Revista Reencuentro, UNAM, n. 40, p. 1-15, México D.F., 2004.

CASTELLS, Manuel. La era de la información. Economía, Sociedad y Cultura. España: Alianza, 1997.

DE KOSTKA FERNÁNDEZ, Estanislao. Políticas públicas. In: Román Reyes (Dir.) Diccionario crítico de Ciencias Sociales, Madrid, Universidad Complutense, 2004. Disponible en: http://www.ucm.es/info/eurotheo/diccionario/P/politicas_ publicas_b.htm.

DELGADO, Adriana. Contribución al análisis de políticas a la formulación y gestión de políticas públicas. Papel Político, n. 14, p. 85-98, 2002.

DE YANZI FERREIRA, Marcel Aspell. La regulación de la cárcel indiana. El caso de Córdoba del Tucumán. Siglo XVIII, In: Anuario, n. 3, CIJS, Centro de Investigaciones Jurídicas y Sociales, Facultad de Derecho y Ciencias Sociales, Universidad Nacional de Córdoba, Córdoba, Argentina, 2002. Disponible en: http://bibliotecavirtual.clacso.org.ar/ar/libros/argentina/cijs/sec3003o.html.

DUNN, William. Public Policy Analysis. An Introduction, 3. ed. New Jersey: Pearson Prentice Hall, 2004.

GARRETÓN, Manuel Antonio. La faz sumergida del iceberg. Estudios sobre la transformación cultural. Santiago, Chile: CESOC, 1994.

GARRETÓN, Manuel Antonio. Social sciences in Latin America. A comparative perspective: Argentina, Brasil, Chile, México and Uruguay. In: GARRETÓN, M. A.; MURMIS, M., DE SIERRA, G.; TRINDADE, H. Social Science Information sur les Sciences Sociales, v. 44, n. 2-3, june-september, Sage Publications, 2005. 
GARRETÓN, Manuel Antonio. Las Ciencias Sociales en Chile. Institucionalización, Ruptura y Renacimiento. In: GARRETÓN, Manuel, A.; DE SIERRA, Gerónimo; MURMIS, Miguel; REYNA, José Luis; TRINDADE, Hélgio (Coord.). Las ciencias sociales en América Latina en perspectiva comparada. México: S. XXI, 2007a.

GARRETÓN, Manuel Antonio. Del post-pinochetismo a la sociedad democrática. Política y sociedad en el bicentenario. Santiago, Chile: Random Mondadori, 2007b.

GIBBONS, M.; NOWOTNY, H.; SCOTT, P. "Mode 2" Revisited: The new production of knowledge - Introduction. Minerva, v. 41, n. 3, Holanda, p. 179-94, 2003.

GIDDENS, A. Vivir en una Sociedad Postradicional. In: BECK, U.; GIDDENS, A.; LASCH, S. Modernización Reflexiva. Madrid: Alianza Universidad, 1997.

GUZMÁN, Virginia; HOLA, Eugenia. El conocimiento como un hecho político. Santiago: CEM, 1996.

HARAWAY, Donna. Ciencia, Cyborgs y Mujeres. Madrid: Ed. Cátedra, 1995.

IHL, O.; KALUSZYNSKI, M.. Pour une sociologie historique des sciences de gouvernement. Revue française d'administration publique, n. 102, p. 229-243, 2002. Disponible en http://www.cairn.info/article.php?ID_REVUE=RFAP\&ID_ NUMPUBLIE $=$ RFAP_102\&ID_ARTICLE $=$ RFAP_102_0229

KRAFT, Michael; FURLONG, Scott. Public Policy: Politics, Analysis and Alternatives. Washington D.C.: CQC Press, 2004.

LINDBLOM, Charles. El proceso de elaboración de políticas públicas. México: MAP, 1991.

LINDBLOM, Charles. Democracia y Sistema de Mercado. México: Fondo de Cultura Económica, 2000.

MENY, Yves; THOENIG, Jean Claude. Las políticas públicas. Barcelona: Ariel, 1992.

MOULIÁN, Tomás. De la política letrada a la política analfabeta. Santiago: LOM, 2004.

OSZLAK, Oscar. ¿ Responsabilización o respondibilidad?: el sujeto y el objeto de un Estado responsable. VIII Congreso Internacional CLAD sobre la Reforma del Estado y de la Administración Pública, Panamá, 28-31, oct., 2003.

RAMOS, Claudio. Cómo Investigan Los Sociólogos Chilenos en los Albores del Siglo XXI: Paradigmas y Herramientas del Oficio. Revista Persona y Sociedad, v. 19, n. 3. Santiago, Chile, p. 85-119, 2005.

URZÚA, Raúl. Reseña de "Qué tipo de nexos para qué tipo de políticas. Estudio comparado en diversos campos de intervención pública en Chile", Grupo GRES- 
$\mathrm{CH}$, Maite de Cea y Manuel Gárate (Ed.), Santiago, Chile: Editorial Universidad Bolivariana, 2006.

VASCONI, Tomás. Las Ciencias Sociales en América del Sur y Chile 1960-1990. Working Paper. Centro de Investigaciones sociales, Universidad ARCIS, 1996.

WEISS, C. H. De Many Meaning of Research Utiization. In: BULNER, M. (Comp.). Social Science and Social Policy. Londres: Allen \& Unwin, 1986, p. 30-40.

\section{Significado de siglas}

CAI: Centros Académicos Independientes.

DIRTRAB: Dirección del Trabajo.

DOS: División de Organizaciones Sociales.

FONDECYT: Fondo Nacional de Desarrollo Científico y Tecnológico.

MIDEPLAN: Ministerio de Planificación y Previsión Social.

MINEDUC: Ministerio de Educación.

MINVU: Ministerio de Vivienda y Urbanismo.

ONG: Organizaciones No Gubernamentales.

SEGPRES: Ministerio Secretaría General de la Presidencia.

SERNAM: Servicio Nacional de la Mujer.

Recebido: $12 / 03 / 2009$

Aceite final: 30/04/2009 